\title{
Warum brauchen wir eine (neue) Bibliothekswissenschaft? Editorial
}

Zusammenfassung: Die Medienschwelle, an der wir uns befinden, stellt viele Institutionen in Frage. Nicht aber die Bibliothek, wie viele äußerst erfolgreiche neue Bibliotheksprojekte (ÖB und WB) belegen. Der Themenschwerpunkt lässt (auch anlässlich der Next Library Conference in Berlin Sept. 2018) unterschiedliche Wissenschaftler zu Wort kommen, die dafür plädieren, sich auch wissenschaftlich mit dem Phänomen Bibliothek (wieder) zu befassen, um besser zu verstehen wie ihre Potentiale den digitalen Wandel positiv begleiten können.

Schlüsselwörter: Bibliothekswissenschaft; Erneuerung; Dataismus; Wissen; soziale Erkenntnistheorie

\section{Why Do We Need Library Science? An Editorial}

Abstract: The turn during the digital transformation is questioning a lot of institutions. But not the Library which seems to be as an institution better off than expected for a long time. This may be seen in the light of recent successful library project either in the pubic sphere as well as in the field of research and academic libraries. The thematic focus collects statements from researchers coming form divers cultures or disciplines arguing for a renewal of Library Science in the light of the digital.

Keywords: Library science; renewal; dataism; knowledge; social epistemology

Welche Rolle könnten Bibliotheken noch spielen im Zeitalter des Dataismus? ${ }^{1}$ Sicher, sie haben immer schon mit Informationen und der Bewältigung der Informationsflut zu tun gehabt. Aber eigentlich waren sie menschenzentriert („,humanistisch“ nach der Definition von Harari) bzw. auf die sie tragende Gemeinschaft oder Gesellschaft ausgerichtet. Ihre Praxis der Strukturierung der Dokumente und Daten in Aufstellung und Katalog ${ }^{2}$ ist nie nur dem reinen Retrieval geschuldet, sondern immer auch der Aufarbeitung des Wissens der Welt als

\footnotetext{
${ }^{1}$ Harari (2017) Kap. 11.

2 Krajewski (2017).
} 
Funktionsgedächtnis wie Aleida Assmann sie charakterisiert. Besonders deutlich wird dies mit dem Beginn der Dokumentationsbewegung im ersten Drittel des 20. Jahrhunderts, ${ }^{3}$ wenn Paul Otlet und Henri Lafontaine unter dem Eindruck der Schrecken des ersten Weltkrieges an der Universal Decimal Classification aber auch an Mundaneum, der "cité mondiale" des Wissens, und der Idee des Völkerbundes arbeiten (wofür Lafontaine später den Friedensnobelpreis erhielt). Der Impetus des Sammelns von Wissen oder zumindest von bibliographischbibliothekarischen Einheiten war nie neutral, sondern immer schon sozial. Die großen amerikanischen Nachkriegsbibliothekare Margeret Egan und Jesse Shera den Kern bibliothekarischer Arbeit schon 1952 als Beitrag zur „sozialen Erkenntnistheorie“ (social epistemology). Und weil sich das „Weltwissen“ auch immer schon sozial konstruiert und „gewachsen“ ist, ${ }^{4}$ ähnlich wie dies ja auch Karl Popper mit seinem Dreiwelten-Konzept postuliert, muss es „gepflegt“ werden - wir würde heute sagen: kuratiert.

Für die Bewältigung der Flut an Informationen - oder besser: Daten - an sich sind jetzt Algorithmen zuständig. Die informationsbasierten Entscheidungen, die wir zunehmend an Maschinen abgeben, brauchen aber immer noch Metadaten und Ontologien, wenn nicht gar Ethiken, die den Informationswert bestimmen. ${ }^{5}$ Im Digitalen entstehen zwei Optionen: entweder ermöglicht die Reduktion der Informationsasymmetrie im Datenkapitalismus die Erstarkung des Marktes in neuer Form oder die Netzwerk- und Feedbackeffekte der neuen Informationstechnologien führen zu starken Zentralisierungen und Monopolisierungen. ${ }^{6}$ Der Dataismus birgt aber auch die Chance, deutlicher sichtbar zu machen, wie komplex die Realität ist und fordert unsere Fähigkeit heraus, die Fülle an Daten für Erkenntnis und Wissen zu nutzen. Und wie Mayer-Schönberger und Ramge es im Schlusswort ihres Buches „Das Digital“ optimistisch formulieren: „Dank Datenreichtum wird unsere Zukunft nicht bloß persönlicher, effizienter und nachhaltiger sein, sondern vor allem gemeinschaftlich - und zutiefst menschlich. ${ }^{\text {" }}$ Sie sagen in ihrer Analyse des „Digitals" allerdings nicht wirklich wie (außer mit ordnungspolitischen Maßnahmen) der nicht mehr geld-, sondern datenbasierte Markt den Sprung zu Erkenntnis, Wissen und Gemeinschaft erreicht. Zu sehr sind die von ihnen selbst thematisierten negativen totalitären, zentralisierenden Tendenzen der Verflachung der Erkenntnis zumindest aktuell stark präsent.

\footnotetext{
3 Hartmann (2012).

${ }^{4}$ Egan und Shera (1952).

${ }^{5}$ Mayer-Schönberger und Ramge (2017) 222.

${ }^{6}$ Ebd. Passim.

${ }^{7}$ Ebd. 227.
} 
Die Gradwanderung wird nicht gelingen ohne spezifische gesellschaftliche Institutionen, die als Intermediäre nicht nur horizontal im Datenmarkt vermitteln (wie Tinder im Heiratsmarkt), sondern auch Vermittlungs- und Katalysatorfunktion übernehmen zwischen der digitalen Datenebene und der analogen (d.h. menschlichen) Erkenntnis- und Wissensebene, z.B. entlang der DIKW Hierarchie. Der Übergang von expliziten Daten zu einem impliziten Wissen ist jedoch, wie Michael Polanyi, der Begründer des Konzepte des „taziten Wissens“ betonte, ${ }^{8}$ zumindest in seiner Umkehrung praktisch nicht möglich (ob wir aus Daten lernen können, war allerdings - zugegeben - nicht sein Thema). Die Beziehung zwischen der Realität und der symbolischen Ebene der Metadaten und bedeutungstragenden Informationen ist ja auch epistemologisch alles andere als klar. ${ }^{9}$ Aber eben daran arbeiten Bibliotheken und Bibliothekare nicht erst seit Kallimachos in Alexandria. Wobei sie sich selten der Mühe unterziehen zu erklären, wie und warum sie es machen.

Wenn denn die Erschließung neuer Datendimensionen die Erkenntnis fördern kann, so ist aber genau diese intermediäre Tätigkeit besonders bedeutsam. Und zwar nicht nur für den Erkenntniszuwachs des Einzelnen, sondern vor allem für die Gemeinschaft - so wir sie denn wollen im Markt. Die Thesen von Mayer-Schönberger und Ramge lassen sich sehr schön an der Open Data- und Citizen-Science-Bewegung verifizieren, aber gerade sie zeigen in der Praxis, dass es Einrichtungen und Orte als Wissenskatalysatoren bedarf wie Open Science Labs, Hackathons, Wissenschaftsläden oder eben Bibliotheken.

An solchen Orten wird nicht im klassischen Sinn Information oder Wissen vermittelt, es sind in erster Linie Orte der Konversation und der Praxis von Gemeinschaften. Der amerikanische Bibliothekswissenschaftler David Lankes hat in seinem kooperativen und preisgekrönten Buch The Atlas of New Librarianship schon 2011 eindringlich darauf hingewiesen, dass wir eine neue Sicht auf Bibliotheken brauchen und genauer hinschauen müssen, was in ihnen nach dem Informationsparadigma eigentlich aktuell geschieht. Als lebendige gesellschaftliche Funktion haben sie sich gewandelt, ohne dass dies von allen wahrgenommen wurde. Interessanterweise nimmt er als Ausgangspunkt die komplexe Theorie des kybernetischen Lerntheoretikers Gordon Pask, ${ }^{10}$ die davon ausgeht, dass Lernen, d.h. die Wissens- und Erkenntnisarbeit („,emergence of knowledge“), stets auf Konversationen zwischen Akteuren beruht und zwar nicht im Sinne des Sender-Empfänger Modells, sondern als gemeinsame „Arbeit am Wissen“.

\footnotetext{
8 Polanyi (1958).

${ }^{9}$ Dreyfus und Talyor (2015).

${ }^{10}$ Vgl. Scott (2001).
} 
Für Lankes folgt im Kern daraus, dass Bibliotheken als Aufgabe haben, der Gemeinschaft durch Förderung der Wissensproduktion zu nützen, in dem sie die Begegnung der Akteure ermöglichen. Das ließe sich selbst im klassischen Konstruktivismus auch von der Autor-LeserBeziehung sagen, von der Intertextualität des Bücherrades oder dem „Geistergespräch“ der Renaissance. ${ }^{11}$ Mit Pask geht er aber darüber hinaus und betont den Aspekt der Community als eigenem sozialen, wissensgenerierenden Element - ohne direkt auf Egan und Shera Bezug zu nehmen. Wissensgenerierung in der Gemeinschaft erfolgt allerdings zu einem großen Ausmaß implizit oder narrativ. ${ }^{12}$ Schon Kallimachos, Zenodot und die Bibliothekare von Alexandria arbeiteten am Narrativ ihrer Weltgesellschaft, indem sie kollaborativ Metadaten schufen. Berti und Costa weisen darauf hin, dass dieser Urmythos einer Bibliothek mitnichten durch einen Brand oder die einsame Entscheidung eines Kalifen verloren ging, sondern durch den technologischen Wandel der zunehmenden Verbreitung des Kodex und die Abnahme des Gedankens der gemeinschaftlichen Erarbeitung des Weltwissens. ${ }^{13}$

Der technologische Wandel, die Medienschwelle von der Bibliosphäre zur Infosphäre, ${ }^{14}$ die wir am Anfang des 21. Jahrhunderts erleben, bedeutet für Bibliotheken, sich bewusst zu machen, was - außer dem Heraussuchen von Information - wirklich an ihren offensichtlich boomenden und gehypten Orten passiert ${ }^{15}$ und wie man sie als „Quelle geistiger Bewegung und neuer Wissensproduktion“16 in der Datenökonomie zum Wohle der Gemeinschaft fruchtbar machen kann.

Dazu reicht es wahrscheinlich nicht, sich mit Informationsverhalten, Information retrieval und Informationsanalyse zu beschäftigen. ${ }^{17}$ Vielmehr müssen Fragen grundlegenderer Natur bearbeitet werden wie die nach der Möglichkeit der Wissensproduktion an diesem Ort oder solche nach der demokratischen Funktion der Institution Bibliothek. Oder die Frage, die Henrik Jochumsen unlängst stellte: ${ }^{18}$,why does nobody care (in terms of research) about such an important institution?“. Die Praxis ist der Wissenschaft ein großes Stück voraus: nicht nur in Skandinavien, den Niederlanden, Katar oder China werden beeindruckende Bibliotheken neu gebaut, die zumindest die Wertschätzung der Gesellschaft gegenüber dieser Institution ausdrücken (oder sich mit ihr schmücken wollen).

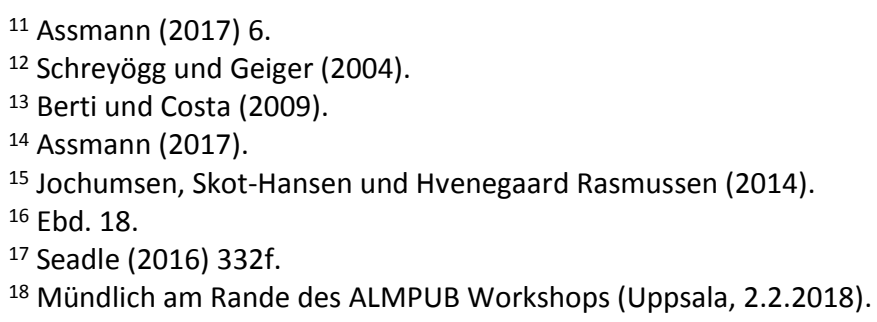


Die hier versammelten Beiträge stellen einen Versuch dar, den Blick zu öffnen auf Diskussionsstränge zur Bibliothekswissenschaft, die sich aus der Praxis des New Librarianship und anderer neuer Ansätze ergeben. Interessant ist dabei zu sehen, dass sich die Autoren oft nicht wirklich kennen (kannten) und doch sogar die selben Bezugspunkte wählen. ${ }^{19}$ Über Sprach- und Disziplingrenzen hinweg werden ähnlich Fragen gestellt und zu einer Erneuerung aufgerufen.

David Lankes beschreibt in seinem Beitrag das Grundkonzept der New Librarianship als notwendige Entwicklung von der Informations- zur Wissensorientierung der Bibliotheken. Dies erfordert mehr als "nur“ Wissensmanagement und genauere Zielgruppenorientierung wie seine Kernbegriffe „facilitating knowledge creation" und „community“ vermuten lassen. Aus dem Wissensmanagement und der Pädagogik wissen wir, dass der Ort und der Raum eine zentrale Rolle spielt für den Wissenstransfer. Begleitend zur Praxis der Entwicklung von DOKK1 in Aarhus entwickelten Kollegen der Royal School of Library and Information Science in Kopenhagen ihr mittlerweile zumindest in Europa weit bekanntes „Four-Spaces“-Modell, ${ }^{20}$ das Henrik Jochumsen in seinem Beitrag bibliothekswissenschaftlich thematisiert. Die beiden aktuell bibliothekswissenschaftlich am meisten diskutierten Konzepte (das von Lankes und das Kopenhagener) sind bisher noch nicht miteinander in Berührung gekommen, obwohl sie m.E. passfähig sind.

Ebenso verdient eine breitere wissenschaftliche Rezeption die französische Diskussion um das Dokument im digitalen Zeitalter. Die Arbeiten sind bekannt geworden unter dem Pseudonym der Arbeitsgruppe des CNRS Réseau thématique pluridisciplinaire „Documents et contenu: création, indexation, navigation“ (RTP-DOC)“ Roger T. Pédauque, der Jean-Michel Salaün (Lyon) angehörte. Er destillierte aus den Ergebnissen das Modell des Dokuments mit seinen drei Dimensionen „vu-lu-su“ heraus, in der die Bibliothek eine zentrale Rolle spielt. Pédauque bezieht sich explizit auf die Tradition der Dokumentation von Paul Otlet und seinen Vorhaben der Wissensstrukturierung. Für Salaün ergibt sich daraus die zentrale Forderung für die Bibliothekswissenschaft, sich erneut mit Informationsarchitektur zu beschäftigen - so wie es z.B. die ASIS\&T schon seit Jahren sogar kommerziell erfolgreich tut. Die Rolle der Bibliothek im Digitalen ist für Salaün ganz zentral die Aufrechterhaltung der Lesbarkeit (,Iu“) der Texte (im abstrakten Barthesschen Sinn) für die Gemeinschaft.

\footnotetext{
${ }^{19}$ Rob Bruijnzeels versicherte mir, dass er Gordon Pask als Bezugspunkt hatte, bevor er David Lankes kennenlernte. ${ }^{20}$ Hvenegaard, Jochumsen und Skot-Hansen (2011).
} 
Ragnar Audunson (Oslo) thematisiert dagegen die in Skandinavien vielfach schon gesetzlich verankerte Rolle von Bibliotheken und anderen Kultureinrichtungen zur Förderung der Demokratie. Hier spielt die Digitalisierung aber auch der Trend herein, dass Bibliotheken zu Erfahrungsorten werden. Wie in anderen praxisorientierten Wissenschaften (z.B. der Gesundheitswissenschaften) gilt es, besonders schnell und reflektiert auf Änderungen in den beruflichen Anforderungen zu reagieren. Die Praxisorientierung bedingt zusätzlich eine hohe Multidisziplinarität und hohes empathisches Abstraktions- und Problemlösungsvermögen.

Ohne Bezug weder auf die amerikanischen noch auf die skandinavischen

Bibliotheksinnovationen hat ja die niederländische Bibliothekswelt schon lange die Tradition der ständigen (erfolgreichen) Erneuerung. Einer ihrer langjährigen Begleiter ist Rob

Bruijnzeels, der hier zusammen mit Joyce Sternheim hinterfragt, ob die aktuellen renommierten Bibliotheksbauten wirklich den neuen Aufgaben der Bibliotheken entsprechen und vor allem, welche Konsequenzen daraus zu ziehen sind für die bibliothekswissenschaftliche Ausbildung.

Aus disziplinärer Außenperspektive nehmen der Literaturwissenschaftler Nikolaus Wegmann und der Medienwissenschaftler Wolfgang Ernst Stellung zur notwendigen Erneuerung der wissenschaftlichen Betrachtung des Phänomens Bibliothek. Während Bibliothekswissenschaftler recht allgemein über ihre longue durée und Nachhaltigkeit sprechen, ${ }^{21}$ geht die Medienwissenschaft direkt in den schmerzenden Kern der Frage und thematisiert Zeitlichkeit von Information vor dem Hintergrund zunehmender Entropie. Sehr verhalten ermahnt Ernst die Bibliothekswissenschaft sich genau damit zu beschäftigen, weil die Medienwissenschaft auf sie angewiesen ist.

Auf der Basis seines immer noch zentralen Buches zur Bibliothek im alexandrinischen Zeitalter „Bücherlabyrinthe“, 22 erinnert Nikolaus Wegmann ebenfalls daran, wie sehr schon viele Geistesgrößen (und nicht nur Goethe) als Bibliothekare gearbeitet und gedacht haben, und dass die Bibliothekswissenschaft immer noch von diesen lernen kann - besonders an der Medienschwelle. Auch er plädiert für einen Neuanfang - wie schon 2000.

Allen Statements gemeinsam ist, dass sie von einer Außenperspektive argumentieren - als Wissenschaftler und nicht als Praktiker. Die aktuelle Situation der extremen Digitalisierung erfordert ein großes Maß an übergreifender Reflexion, eine Art archimedische Position, die

\footnotetext{
${ }^{21}$ Vodosek (2016).

22 Amerikanische Übersetzung in Vorbereitung.
} 
uns hilft, eine klare Sicht auf die Entwicklungen nicht nur unserer Institution zu erhalten, um nicht im Alarmismus oder Defaitismus zu versinken. Der historische Blick und der Blick von außen ist dazu meist hilfreich. Wichtig erscheint aber auch eine wissenschaftliche Neugründung der Fachdisziplinen - vielleicht unter ganz anderen Voraussetzungen. Viele internationale Indizien deuten darauf hin, dass wir unsere Institution und Sichtweise nicht den Dataisten überlassen sollten.

\section{Literaturverzeichnis}

Assmann, Aleida (2017): Das kulturelle Gedächtnis zwischen materiellem Speicher und digitaler Diffusion. In: Michael Knoche (Hg.): Die Zukunft des Sammelns an wissenschaftlichen Bibliotheken. Wiesbaden: Harrassowitz Verlag (Bibliothek und Wissenschaft, 50) 1-18.

Berti, Monica; Costa, Virgilio (2009): The Ancient Library of Alexandria. A Model for Classical Scholarship in the Age of Million Book Libraries. Preprint of a lecture. International Symposium on the Scaife Digital Library (held at the VisCenter of the University of Kentucky). Lexington, KY, 13.03.2009. Online verfügbar unter http://www.perseus.tufts.edu/publications/Berti-Costa_Alexandria_Kentucky.pdf, zuletzt geprüft am 20.02.2018.

Dreyfus, Hubert L.; Taylor, Charles (2015): Retrieving realism. Cambridge, Mass. [u.a.]: Harvard Univ. Press.

Egan, Margeret E.; Shera, Jesse H. (1952): Foundations of a Theory of Bibliography. In: Library Quarterly, 22 (2) 125-37.

Harari, Yuval Noah (2017): Homo Deus. Eine Geschichte von Morgen. München: C.H.Beck.

Hartmann, Frank (2012): Die Logik der Datenbank. Zwischen Leibniz und Google - Otlet der Weltbibliothekar. In: Frank Hartmann (Hg.): Vom Buch zur Datenbank. Paul Otlets Utopie der Wissensvisualisierung. Berlin: Avinus-Verl. (Forschung visuelle Kultur, 2) 11-63.

Hvenegaard, Casper; Jochumsen, Henrik; Skot-Hansen, Dorte (2011): Biblioteket i byudviklingen. Oplevelse, kreativitet og innovation. Kopenhagen: Danmarks Biblioteksforening; Det Informationsvidenskabelige Akademi.

Jochumsen, Henrik; Skot-Hansen, Dorte; Hvenegaard Rasmussen, Casper (2014): Erlebnis, Empowerment, Beteiligung und Innovation: Die neue Öffentliche Bibliothek. In: Olaf Eigenbrodt und Richard Stang (Hg.): Formierungen von Wissensräumen. Optionen des Zugangs zu Information und Bildung. Berlin: De Gruyter Saur (Age of access? - Grundfragen der Informationsgesellschaft, 3) 67-80.

Krajewski, Markus (2017): Tell Data from Meta. Tracing the Origins of Big Data, Bibliometrics, and the OPAC. In: Osiris, 32 (1) 224-40. DOI: 10.1086/694228.

Lankes, R. David (2011): The atlas of new librarianship. Cambridge, Mass: MIT Press. 
Mayer-Schönberger, Viktor; Ramge, Thomas (2017): Das Digital. Markt, Wertschöpfung und Gerechtigkeit im Datenkapitalismus. Berlin: Econ.

Polanyi, Michael (1958): Personal knowledge. Towards a post-critical philosophy. London: Routledge \& Kegan.

Popper, Karl (1978): Three Worlds. The Tanner Lecture on Human Values. Tanner Humanities Center. University of Michigan, 07.04.1978.

Schreyögg, Georg; Geiger, Daniel (2004): Kann implizites Wissen Wissen sein? Vorschläge zur Neuorientierung im Wissensmanagement. In: Boris Wyssusek (Hg.): Wissensmanagement komplex. Perspektiven und soziale Praxis. Berlin: Schmidt, 43-54.

Scott, Bernard (2001): Gordon Pask's Conversation Theory. A Domain Independent Constructivist Model of Human Knowing. In: Foundations of Science, (6) 343-60.

Seadle, Michael (2016): Fragility and the Future of Library Ecuation. In: A. Degkwitz (Hg.): Bibliothek der Zukunft - Zukunft der Bibliothek. Festschrift für Elmar Mittler anlässlich seines 75. Geburtstages. Berlin: De Gruyter, 312-33.

Vodosek, Peter (2016): Die Entdeckung der Nachhaltigkeit. Ein bibliothekshistorischer Rückblick. In: A. Degkwitz (Hg.): Bibliothek der Zukunft - Zukunft der Bibliothek. Festschrift für Elmar Mittler anlässlich seines 75. Geburtstages. Berlin: De Gruyter, 334-53.

Wegmann, Nikolaus (2000): Bücherlabyrinthe. Suchen und Finden im alexandrinischen Zeitalter. Köln u.a.: Böhlau.

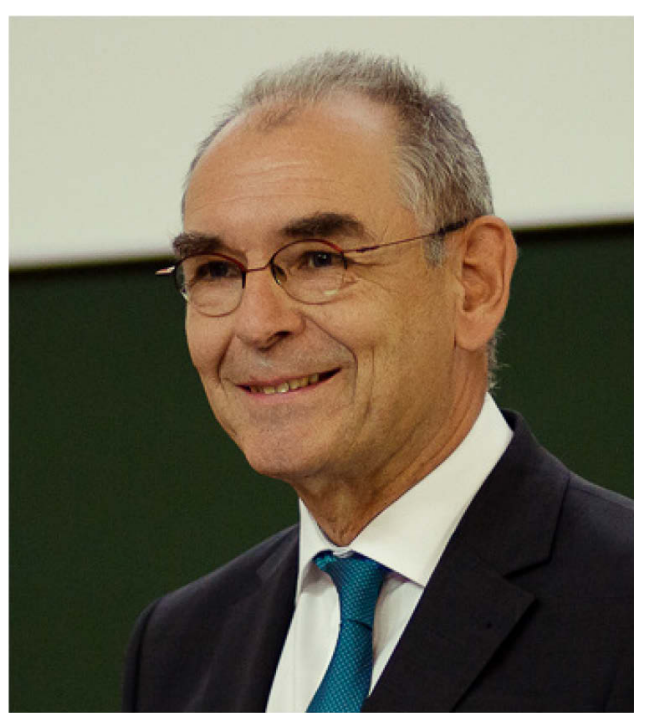

Prof. Dr. Hans-Christoph Hobohm

Bibliotheks- und Informationswissenschaft

Fachhochschule Potsdam

Kiepenheuerallee 5

D-14469 Potsdam

hobohm@fh-potsdam.de 